\title{
Evangelizar desde las redes sociales: el caso de los cardenales en Twitter
}

Recibido: 19 de abril de 2013

Jesús A. PÉREZ DASILVA

Universidad del País Vasco (UPV-EHU)

jesusangel.perez@ehu.es

María Teresa SANTOS DÍEZ

Universidad del País Vasco (UPV-EHU)

mariateresa.santos@ehu.es

Aceptado: 13 de octubre de 2013

\begin{abstract}
Resumen
Aprovechando la renuncia de Benedicto XVI se ha estudiado la actividad en Twitter de los 117 cardenales electores que se han reunido en el Cónclave para elegir al nuevo Papa. La hipótesis de partida es que, en un contexto de distanciamiento creciente de la población, los principales miembros de la Iglesia Católica se valen de tecnologías como la red de microblogging porque, entre otras cosas, aumentan su visibilidad, reducen el coste de las acciones de evangelización, favorecen la cooperación entre los fieles y facilitan la difusión de información religiosa. En este artículo se ve que predomina el modelo comunicativo ‘top-down' y que, salvo contadas excepciones, los cardenales tienden a usar la herramienta más como una plataforma complementaria de difusión que como un espacio de conversación y escucha.

Palabras clave: información religiosa, evangelización, redes sociales, Twitter, Iglesia Católica
\end{abstract}

\section{Evangelizing from social networks: the case of the Cardinals on Twitter}

\begin{abstract}
Taking advantage of the unexpected resignation of Benedict XVI, this study examined the Twitter activity of 117 cardinal electors who have gathered in the 2013 conclave to elect a new pope. The hypothesis is that, in a context of decreasing people who pray and go to church, leading members of the Catholic Church make use of technologies such as microblogging network because, among other things, increase visibility, reduce the cost of evangelization, promote cooperation between the faithful and facilitate the dissemination of religious information. In this article we can see that the predominant communicative model is 'topdown' and that, with few exceptions, the Cardinals (a group with a mean age of 77 years) tend to use the tool as a complementary platform rather than a space of conversation and listening.
\end{abstract}

Keywords: religious information, evangelization, social networks, Twitter, Catholic Church

\section{Referencia normalizada}

PÉREZ DASILVA, Jesús A. y SANTOS DÍEZ, María Teresa (2014): "Evangelizar desde las redes sociales: el caso de los cardenales en Twitter". Estudios sobre el Mensaje Periodístico. Vol. 20, Núm. 1 (enero-junio), págs.: 211-227. Madrid, Servicio de Publicaciones de la Universidad Complutense.

Sumario: 1. Introducción. 2. Metodología. 3. Resultados; 3.1. Presencia; 3.2. Potencial de influencia; 3.3. Actividad; 3.4. Interacción; 3.5. Reacciones generadas en los usuarios. 4. Conclusiones. 5. Referencias bibliográficas.

\section{Introducción}

Los nuevos medios de comunicación se convierten en un instrumento de innegable valor para la difusión del Evangelio y la transmisión de valores por parte de la Iglesia católica. Para autores como Cordero las nuevas tecnologías plantean grandes oportunidades si se aprovechan los aspectos positivos, de modo que Internet "se está mostrando como una herramienta crucial para la captura y circulación de informaciones cristianas y de vida religiosa" (Cordero, 2008: 85). La combinación de la Red con la religión está dando lugar a una manera distinta de difundir el Evangelio y la fe 
a las personas alejadas de la Iglesia mediante la emisión de contenidos religiosos a través de Internet. En este sentido, en palabras del Papa Benedicto XVI:

«Entre las nuevas formas de comunicación de masas, hoy se reconoce un papel creciente a Internet, que representa un nuevo foro para hacer resonar el Evangelio, pero conscientes de que el mundo virtual nunca podrá reemplazar al mundo real, y que la evangelización podrá aprovechar la realidad virtual que ofrecen los new media para establecer relaciones significativas sólo si llega al contacto personal, que sigue siendo insustituible. En el mundo de Internet, que permite que millones y millones de imágenes aparezcan en un número incontable de pantallas de todo el mundo, deberá aparecer el rostro de Cristo y oírse su voz, porque "si no hay lugar para Cristo, tampoco hay lugar para el hombre"» (Benedicto XVI, Ex. Ap. Verbum Domini, n. 113).

A pesar de que el tema religioso se puede considerar que pertenece al ámbito privado, está lejos de desaparecer y continúa estando presente. (Beckford 2003; Williams 2003). Según autores como Mancilla afecta a "en grados diversos todos los ámbitos de sus vidas, o que, por lo menos, establecen conexiones entre el ámbito privado y algunas cuestiones sociales o políticas" (Mancilla: 2011). Por ello, no resulta extraño que la Iglesia católica (Caravaca, 2013) haya optado por aprovechar las posibilidades que ofrece Internet para llevar a millones de personas la palabra de Dios. Por ello no resulta extraño que surjan iniciativas en la red innovadoras como es el caso del rezo del rosario en la web www.mirezo.com, que desde 2007 posibilita rezar a los creyentes cristianos de cualquier parte del mundo. También, se pueden encender velas desde el ordenador en la Iglesia de la Anunciación de Nazaret de Israel. Más cerca, la Catedral de Santiago de Compostela (diario 20 Minutos, 2010) incorpora desde 2010 lampadarios virtuales a través de la página web mivela.com.

El propio Pontífice Benedicto XVI, ante la importancia de las redes sociales, en diciembre de 2012 abrió una cuenta en Twitter con una finalidad evangelizadora. Según el Obispo P. José Ignacio Munilla', a partir de este momento se produjo un efecto llamada, que ayudó mucho a los que ya estaban en la Red. La decisión del Papa estuvo ligada al deseo de la Iglesia por estar allí donde está el hombre, y el hombre hoy día está en las redes sociales. Precisamente, se ha pasado en 15 años de 10 millones a 2.100 millones de personas online en el mundo (TNS, 2012: 11). La Red se ha convertido en parte fundamental de nuestras vidas y, especialmente, los medios sociales como las redes sociales, los blogs o los foros. La media de horas, a nivel global, que pasamos a la semana en Internet es de 18 horas, con el 18\% de ese tiempo dedicado a redes sociales (TNS, 2012: 15). En España, la penetración es del 67,1\%, lo que supone la existencia de 23,3 millones de internautas, de los que 16,6 millones de personas (el 71,4\%) usan Internet diariamente (véase informe SIE 2012 de Telefónica). Su importancia es tal que un 50\% de los estudiantes universitarios y el $48 \%$ de los jóvenes profesionales españoles en activo consideran Internet un recurso tan vital como el aire, el agua, la comida o la vivienda. Según un informe realizado por Cisco en 2011, si tuvieran que elegir entre disfrutar de conexión a Internet o tener un vehículo, el $67 \%$ de los universitarios españoles elegiría Internet.

${ }_{1}$ Programa La Tarde de la COPE. 21/02/2013. 
Por lo tanto, no resulta extraño el debut del Sumo Pontífice en Twitter (@pontifex) a finales del 2012. Su primer 'tuit' consiguió en 8 horas más de 83.000 'retuits' (ABC, 2012). Un mes después, un estudio de la agencia $\operatorname{Oog}^{2}$ para la revista Popoli de los jesuitas italianos (véase Mújica, 2013) recoge que se habían generado 270.456 respuestas de los que 200.000 eran neutros y 26.426 eran positivos, destacando con un $26 \%$ el reenvío de los mensajes del Papa, los agradecimientos y los buenos deseos $(25 \%)$ y la petición de oraciones $(16,6 \%)$. Aunque menos numerosos que los positivos, también se contabilizaron 22.542 mensajes negativos referentes a temas como los casos de pedofilia en la Iglesia (26\%) o las críticas al poder y a la riqueza del Vaticano $(20 \%)$.

Según Pou (2008:1): "las creencias, las prácticas de fe y la vida de las comunidades religiosas constituyen una realidad compleja cuya presencia en los medios de comunicación no siempre es satisfactoria", ya que no responde a las necesidades actuales de amplios sectores de la población. En este sentido, los medios sociales se convierten en una nueva forma de llegar a los fieles en un momento de crisis en que escasean las vocaciones y la religión católica pierde influencia en la sociedad. Según se desprende de estudios del CIS, en 2008 el 15\% se declaraban católicos no practicantes, mientras que en 2013 el porcentaje se incrementa hasta el 59,4\%. En esta línea, los que dicen ir a misa los domingos se reducen a un $12,4 \%$, cifra que baja a un $6 \%$ entre los menores de 30 años. Los dirigentes eclesiásticos saben que las TIC hoy día son útiles para resucitar creencias adormecidas y no dudan en hacer uso de ellas.

Pero esta apuesta personal del Papa por estar donde están sus fieles, forma parte de una estrategia más amplia que engloba a toda la Institución. El púlpito se sustituye por las redes, foros y bitácoras aunque el contenido es el mismo. Como afirmaba el padre Munilla, director de Radio María, en el Congreso Mundial de Radios Católicas celebrado entre el 19 y 21 de junio de 2008 :

"Si en sus comienzos no fue precisamente fácil, hoy, con mas medios, tenemos también que intentar que se escuche la voz del Señor en todos los rincones de España, en todos los ámbitos, no sólo en el católico, sino llevarlo también a todas la personas que desconocen la vida de la Iglesia y que la conozcan también a través de la radio, de la televisión, a través de todos los medios que están hoy en día al alcance de la Iglesia Católica" (RTVD, 2008)

Además del Santo Padre, hoy día en las redes sociales también están presentes sacerdotes como el jesuita Antonio Spadaro (@antoniospadaro), director de la revista Civilitá Cattólica, que es el más antiguo en Twitter (creo su perfil en mayo de 2007) y que tiene un índice Klout (mide el grado de influencia en las redes sociales) de 73. También hay que mencionar otras iniciativas como el perfil @CurasOnline y el dominio www.curasonline.com (Caravaca, 2013). También los cardenales han desembarcado en la Red y han descubierto las ventajas de interactuar con los fieles en el ciberespacio. Por ejemplo, como se puede ver en la parte final de este estudio, algunos ya emplean Twitter para inspirar a sus fieles mediante citas famosas, invitaciones a tener a Dios presente, defender los valores cristianos o apoyar campañas contra el aborto o el matrimonio gay.

${ }^{2}$ El estudio se centró en la cuenta en inglés @pontifex. 
En el caso de Facebook, el Cardenal Antonio Tagle de Manila destaca por encima de los demás con más de 120.000 seguidores. A gran distancia le sigue el cardenal americano Timothy Dolan (Nueva York) con cerca de 20.000 seguidores. Pero estas plataformas también son empleadas por los fieles para combatir los agravios que sufren y mejorar su mundo. Como apuntan Tascón y Quintana (2012: 9) "se extiende por el mundo una nueva forma de activismo social" y un ejemplo es lo ocurrido a comienzos de 2013, mientran se escribían estas líneas, cuando más de 8.500 personas se 'dirigieron' a Facebook protestando por el cierre el 25 de enero de una página católica de catequesis que contaba con 115.000 seguidores (ACI, 2013). El cierre fue interpretado como un ataque contra la religión y puso en un aprieto a la red de Zuckerberg. Y es que la tecnología cambia las dinámicas de la movilización social. La democratización de la tecnología hace posible un 'ciberactivismo' basado en las multitudes conectadas que en momentos críticos se pueden convertir en activistas.

\section{Metodología}

Aprovechando la inesperada dimisión de Benedicto XVI y la elección de un nuevo Papa, este estudio ha examinado la presencia en Twitter de los 117 cardenales electores que se han reunido para elegir al nuevo Sumo Pontífice. Una población objeto de estudio a la que se le ha añadido también el perfil del Santo Padre. Se ha optado por Twitter porque, según informes como el de la consultora Cocktal Analysis (2012: 9), ha duplicado su penetración hasta llegar a ser la segunda red social. En la misma línea, el informe SIE de la Fundación Telefónica (2012: 84-86) señala que es la tercera entre las plataformas que tienen un mayor uso por parte de los internautas y la que tiene el mayor porcentaje $(12,2 \%)$ de usuarios conectados continuamente. Hay que tener en cuenta que cuando Benedicto XVI fue elegido en 2005, la red de microblogging todavía no existía, y que una de las novedades de este Cónclave de 2013 es el éxito mundial de dicha plataforma y la presencia creciente en la misma de destacados miembros de la Institución.

La hipótesis de partida es que los principales miembros de la Iglesia Católica se valen de las TIC porque, entre otras cosas, amplifican el mensaje cristiano, reducen el coste de las acciones de evangelización, favorecen la cooperación entre los fieles y facilitan la difusión de información religiosa en general. En un contexto de distanciamiento creciente de la población, la plataforma Twitter se convierte también en una herramienta útil para llegar a las personas alejadas de la Iglesia y difundir el Evangelio. Por ello, el estudio ha tratado de responder a los siguientes interrogantes: ¿Cuál es la presencia de los príncipes de la Iglesia en la red de microblogging? ¿Quiénes son los cardenales más seguidos y más influyentes? ¿Cómo se construyen sus relaciones sociales? ¿Qué contenidos priman en los perfiles de dichos usuarios?

Para dar respuesta a dichas cuestiones se ha elaborado en primer lugar una ficha de análisis con 18 indicadores que ha seguido las pautas de cibermetría propuestas, entre otros, por Codina (2003) y Alonso, Figuerola y Zazo (2004). En segundo lugar, todos los perfiles encontrados han sido examinados aplicando las siguientes herramientas de análisis online: Twerpscan, Tweetstat, Twitalyzer, Tweeteffect, Twittercounter, Retweetrank y Tuitonomy. Dichas aplicaciones funcionan en base a algoritmos que aseguran 
la validez científica de los datos obtenidos con niveles de confianza que no bajan del $95 \%$ y un error muestral inferior al $+/-5 \%$. En total, se han analizado todos los mensajes publicados en todas las cuentas creadas por los cardenales, que van desde el primer perfil abierto el 13 de mayo de 2009 (correspondiente a su eminencia Sean O'Malley) hasta las 15:00 horas del 1 de abril de 2013 (Lunes de Pascua). En total, han sido examinadas 6.789 publicaciones más 9 correspondientes al perfil de Francisco I.

Las variables han sido las siguientes:

1. Presencia: Se han buscado los perfiles en Twitter de los 117 cardenales electores más el del Papa. Para la medición de este parámetro se ha elaborado un apartado en la ficha de análisis que permite una respuesta binaria (Sí/No). En su diseño, como en toda la ficha, se han seguido las pautas de cibermetría propuestas por Alonso, Figuerola y Zazo (2004).

2. Creación: Se han examinado todos los perfiles encontrados y se ha retrocedido hasta descubrir el primer 'tuit' de cada una de las cuentas. Se han recogido los datos de participación en la red mencionada hasta el 1 de abril de 2013 a las 15:00 horas.

3. Seguidores: Número de cuentas en la red de microblogging suscritas a la cuenta de un cardenal que leen sus mensajes.

4. Personas a las que sigue: Perfiles en Twitter que son objeto se seguimiento por parte de un cardenal por considerarlas de interés. Lista de personas a las que lee.

5. Mensajes: Número de publicaciones desde la creación del perfil. Este dato junto a la 'media diaria' son los indicadores que sirven para medir la actividad en Twitter.

6. Media diaria: Número de 'tuits' de media que publican al día. Guarda relación con las publicaciones totales de un perfil. Cuanto más alto es este número más activo es el usuario en Twitter.

7. Relación seguidores/siguiendo: Ratio de seguidores por cada persona a la que siguen. Un número mayor de 1 significa que el usuario tiene más seguidores que gente a la que sigue. Un ratio alto combinado con una base amplia de seguidores es un buen indicador de que el usuario es influyente.

8. Listas/1.000 usuarios: La media de personas que añadieron al usuario a una lista pública (por cada 1.000 seguidores). Un número alto combinado con una base amplia de seguidores significa que los tweets del usuario son considerados de especial relevancia por terceros.

9. Retweets: Porcentaje de 'retuits' en el total de mensajes analizados. Cuanto más alto es este valor más interactúa el usuario con terceros. Este indicador, junto a los indicadores 'menciones' y 'respuestas' nos permite medir el grado de interacción de un cardenal.

10. Menciones: Media del número de menciones por tweet. Cuanto más alta sea esta media más interacción tiene el usuario con terceros.

11. Respuestas: Porcentaje de respuestas en el total de mensajes analizados. Sirve también para medir la interacción ya que cuanto más alto es este indicador más interacción genera un usuario.

12. Enlaces: Media de enlaces incluidos en cada 'tuit'. Cuanto más alto es este número más probable es que el usuario sea una fuente de información para otros.

13. Hashtags: Media de hashtags por mensaje. Cuanto más alto es este indicador más probable es que los tweets de un usuario sean encontrados en una búsqueda. 
14. Mensajes 'retuiteados' por terceros: Proporción de los tweets de un usuario que son 'retuiteados' por otros. Un valor alto significa que genera una reaccción positiva en los usuarios y que el cardenal es considerado como una fuente valiosa de información por terceros. Este indicador guarda estrecha relación con el número de veces que dichos 'tuits' son 'retuiteados' (véase variable $\mathrm{n}^{\mathrm{o}} 15$ ).

15. Frecuencia en que los mensajes son 'retuiteados' por terceros: Media de 'retuits' o número de veces en que los mensajes de un usuario han sido 'redifundidos' por terceros'. Un valor alto significa que el cardenal es considerado como una fuente valiosa de información por terceros (se complementa con la variable $\mathrm{n}^{\circ} 14$ ).

16. Día y hora: Recoge el momento en que un usuario publica sus mensajes.

17. Plataforma: Se examina la herramienta empleada para publicar los mensajes. Por ejemplo, la página de Twitter, la aplicación para IPhone, para el IPad, etc.

Por último, se quiere hacer hincapié en que este estudio profundiza en una línea de investigación de candente actualidad, debido a la elección de Francisco I, como son los contenidos religiosos y los nuevos medios en la Red. La faceta analizada -la de los cardenales en la red de microblogging- ha tenido gran impacto y ha sido objeto diario de conversación a comienzos de 2013 en la opinión pública y en los medios de comunicación (véase la prohibición para que los cardenales electores utilizasen Twitter durante el cónclave en los periódicos de mayor tirada). Para este artículo se han examinado aportaciones como las de Sánchez Redondo (2001), Rojo (2006), Gutiérrez y Huertas (2007), Gómez García (2009), Pou Amérigo (2008), Mancilla (2011), Tascón (2012) o Pérez-Latre (2012) entre otros. También se han consultado diferentes estudios hechos en el extranjero como sobre canales de comunicación alternativos como los de Gueorguieva (2008); Gil de Zúñiga (2009); Baumgartner y Morris (2010); Sudulich (2010); Vergeer, Hermans y Sams (2010). También han resultado de especial interés When religión meets new media de Heidi Campbell (2010) y el de Baesler (2013) sobre religión, móviles, Facebook y e-mail.

\section{Resultados}

\subsection{Presencia}

En lo que se refiere a la presencia en la Red, el rastreo efectuado entre enero y marzo de 2013 permite concluir que ya son 14, de un total de 117 cardenales electores, los que disponen de un perfil activo en Twitter. Se ha contabilizado también el perfil del cardenal Scola (@angeloscola) a pesar de que se dio de baja justo al empezar el Cónclave de 2013. Serían 15 si incluimos la cuenta del Papa (@pontifex) creada a finales del 2012. Este es un dato significativo del esfuerzo de adaptación a los nuevos tiempos que está llevando a cabo la Iglesia, ya que es un colectivo que tiene una media de edad de 77 años (Bedoya, 2012) lo que lo coloca en la franja de edad menos entusiasta de los adelantos tecnológicos. Ya que según el informe SIE de la Fundación Telefónica (2012: 25), la utilización de las redes sociales para comunicarse está muy vinculada a la edad de las personas y son los menores de 35 años (entre 16 y 24 años suponen un 54,1\% y entre 25 y 34 años suponen un $28,8 \%$ ) los que más emplean las redes sociales como herramienta de comunicación. Por el contrario, el grupo de mayores de 65 años es el que menos utiliza las redes sociales con un $0,8 \%$. A la luz de estos datos, un vistazo a la web del colegio cardenalicio (donde aparecen ordenados por la edad, entre otras variables) revela que el más joven es el cardenal de la India 
Baselios Cleemis Thottunkal nacido el 15 de junio de 1959 y que el $81,2 \%$ de los electores han nacido antes de 1945, lo que muestra que 95 de los cardenales tienen más de 68 años. Una edad bastante alta que en teoría los sitúa dentro de la franja menos proclive al uso de las redes sociales. Por eso es positivo comprobar que 14 de ellos (12\%) han incorporado Twitter dentro de su estrategia de comunicación.

El primero en dar el salto a la red de microblogging fue el cardenal Sean O'Malley (Boston,@CardinalSean) el 13 de mayo de 2009. Nueve días después lo hizo el cardenal de Milán Angelo Scola (@angeloscola).El resto, en cambio, se fue incorporando paulatinamente a lo largo del 2011 y del 2012. Según Pérez-Latre, en el desembarco en la Red resulto trascendental la aparición del nuevo portal news.va el 29 de junio de 2011, que integraba las plataformas de comunicación 2.0 (Twitter, Facebook y YouTube). De este modo, el Vaticano entraba de lleno en la comunicación digital. (Los medios y la religión en el contexto de la nueva evangelizacion. La sorpresa vino de la mano del Santo Padre (@pontifex) cuando en diciembre de 2012 escribió su primer 'tuit': "Queridos amigos me uno a vosotros con alegría por medio de Twitter. Gracias por vuestra respuesta generosa. Os bendigo a todos de corazón". Su éxito fue inmediato, superando los 83.000 'retuits' si se suman las ocho cuentas oficiales que tiene en distintos idiomas. El 28 de febrero de 2013, tras la renuncia de Benedicto XVI, todos los mensajes de la cuenta fueron borrados. El perfil fue activado por Francisco I el 17 de marzo y mientras se escribe este artículo ha publicado ya nueve mensajes.

Tabla 1: Cardenales electores con cuenta en Twitter. Fuente: elaboración propia

\begin{tabular}{|l|c|l|}
\multicolumn{1}{c}{ Cardenal } & Creación & \multicolumn{1}{c|}{ Apodo } \\
\hline Roger Mahony & $31 / 01 / 2012$ & @CardinalMahony \\
\hline Angelo Scola & $22 / 05 / 2009$ & @angeloscola \\
\hline Sean O'Malley & $13 / 05 / 2009$ & @CardinalSean \\
\hline Gianfranco Ravasi & $09 / 06 / 2011$ & @CardRavasi \\
\hline Odilon Scherer & $05 / 07 / 2011$ & @DomOdiloScherer \\
\hline Wilfrid Napier & $08 / 07 / 2011$ & @CardinalNapier \\
\hline Lluís Martínez Sistach & $12 / 03 / 2012$ & @sistachcardenal \\
\hline Timothy Dolan & $30 / 05 / 2012$ & @CardinalDolan \\
\hline Ruben Salazar & $07 / 12 / 2012$ & @cardenalruben \\
\hline Jesús López Rodríguez & $05 / 01 / 2012$ & @CardenalLopez \\
\hline Donald Wuerl & $15 / 06 / 2011$ & @Cardinal_Wuerl \\
\hline Peter Turkson & 2013 & @TurksonCardinal \\
\hline Norberto Rivera & $09 / 02 / 2011$ & @primadodemexico \\
\hline Luis Antonio Tagle & $25 / 11 / 2011$ & @AntonioTagle \\
\hline \multicolumn{1}{|c|}{ Papas } & Creación & Apodo \\
\hline Benedicto XVI & $12 / 12 / 2012$ & @pontifex \\
\hline Francisco I & $17 / 03 / 2013$ & @pontifex \\
\hline
\end{tabular}

\subsection{Potencial de influencia}

Cada vez más gente sigue estas cuentas en la red de microblogging. El lider indiscutible es el perfil del Papa. Si Benedicto XVI superaba los 2,5 millones entre sus ocho cuentas, Francisco I tiene ya solo en la cuenta principal 2.254.469 seguidores. Entre los 
cardenales, la estrella es el arzobispo de Nueva York Timothy Dolan (@CardinalDolan) con 112.000 incondicionales, cantidad que dobla a la de su inmediato perseguidor, el italiano Gianfranco Ravasi, presidente del Pontificio Consejo de la Cultura, (@CardRavasi), que apenas llega a los 50.000. Aunque además de estos dos, existen otros tres que superan los diez mil partidarios: el cardenal brasileño Odilon Scherer, el arzobispo de Milán Angelo Scola y el cardenal capuchino americano Sean O'Malley. Este último destaca además por ser el cardenal que más personas sigue (692) lo que indica que hay muchas cuentas que le resultan atractivas y que sigue sus conversaciones.

Resulta de interés examinar también la métrica seguidos/seguidores ya que un ratio alto (un número mayor a 1) combinado con una base amplia de seguidores es un buen indicador de que el usuario es influyente. Aquí destacan los cardenales Timothy Dolan y Ruben Salazar porque se da el caso de que solo siguen la cuenta del Papa. En tercer lugar aparece el italiano Gianfranco Ravasi con 993 seguidores por cada usuario al que sigue. En este sentido también es atractivo estudiar la métrica 'Listas/1.000 usuarios'. Aquí se sitúa en primer lugar el arzobispo de Washington Donald Wuerl con 50 puntos, seguido del arzobispo de Barcelona Lluís Martínez Sistach con 39 y del de Los Ángeles Roger Mahony con 37. Su valor radica en que señala la media de cibernautas que se han tomado el tiempo y la molestia de clasificar el perfil en una lista pública (por cada 1.000 seguidores) lo que es indicativo de la importancia del mismo en una temática concreta ${ }^{3}$. Un número alto combinado con una base amplia de seguidores significa que los tweets del cardenal son considerados de especial relevancia por terceros.

Tabla 2: Número de seguidores. Fuente: elaboración propia

\begin{tabular}{|l|r|r|}
\hline \multicolumn{1}{|c|}{ Cardenal } & \multicolumn{1}{c|}{ Seguidores } & \multicolumn{1}{c|}{ Siguiendo } \\
\hline Timothy Dolan & 111.854 & 1 \\
\hline Gianfranco Ravasi & 48.643 & 49 \\
\hline Odilon Scherer & 35.792 & 123 \\
\hline Angelo Scola & 20.015 & 39 \\
\hline Sean O'Malley & 18.754 & 692 \\
\hline Wilfrid Napier & 8.892 & 68 \\
\hline Ruben Salazar & 8.617 & 1 \\
\hline Roger Mahony & 5.951 & 6 \\
\hline Lluís Martínez Sistach & 5.548 & 25 \\
\hline Norberto Rivera & 4.706 & 31 \\
\hline Jesús López Rodríguez & 4.467 & 11 \\
\hline Luis Antonio Tagle & 3.605 & 88 \\
\hline Donald Wuerl & 2.697 & 5 \\
\hline Peter Turkson & & - \\
\hline \multicolumn{1}{|c|}{ Papas } & Creación & Apodo \\
\hline Francisco I & & \\
\hline
\end{tabular}

3 Que alguien te clasifique en una lista en Twitter es un reconocimiento al valor de tus aportaciones. 
Tabla 3: Potencial de influencia. Fuente: elaboración propia

\begin{tabular}{|l|r|r|}
\hline \multicolumn{1}{|c|}{ Cardenal } & $\begin{array}{c}\text { Seguidores / } \\
\text { Seguidos }\end{array}$ & $\begin{array}{c}\text { Listas / 1.000 } \\
\text { seguidores }\end{array}$ \\
\hline Donald Wuerl & 539 & 50 \\
\hline Lluís Martínez Sistach & 222 & 39 \\
\hline Roger Mahony & 992 & 37 \\
\hline Norberto Rivera & 152 & 34 \\
\hline Wilfrid Napier & 131 & 33 \\
\hline Sean O'Malley & 27 & 26 \\
\hline Ruben Salazar & 8.617 & 23 \\
\hline Jesús López Rodríguez & 406 & 23 \\
\hline Luis Antonio Tagle & 41 & 20 \\
\hline Angelo Scola & 513 & 17 \\
\hline Gianfranco Ravasi & 993 & 14 \\
\hline Odilon Scherer & 291 & 11 \\
\hline Timothy Dolan & 111.854 & 10 \\
\hline Peter Turkson & - & - \\
\hline
\end{tabular}

\subsection{Actividad}

Pero la influencia no depende solo de la métrica 'número de seguidores', sino del número de esos partidarios que leen y actúan en el sentido de la recomendación. Un número alto, a partir de ciertas cantidades, lo único que da es una idea aproximada del grado de influencia de un perfil. Como explica el cofundador de Twitter, Evan Williams, esta métrica se instauró para determinar la importancia de un usuario ${ }^{4}$ pero es sobre todo un indicativo del potencial de atención que tiene una cuenta. Por lo tanto, para fidelizar usuarios y aprovechar el perfil para alcanzar un nivel de influencia hay que estar activo y ser constante 'tuiteando'. Generar contenido actualizado es la mejor manera de darse a conocer y la forma más sencilla de medir la actividad es a través del número de mensajes publicados. En este apartado los cardenales más dinámicos son el brasileño Odilon Scherer, el español Lluís Martínez Sistach, los italianos Gianfranco Ravasi y Angelo Scola, y el sudafricano Wilfrid Napier. Todos publican como mínimo un 'tuit' al día y como máximo tres mensajes diarios (es el caso del cardenal Scherer).

Tabla 4: Media diaria de mensajes. Fuente: elaboración propia

\begin{tabular}{|l|r|r|}
\multicolumn{1}{|c|}{ Cardenal } & \multicolumn{1}{c|}{ Mensajes } & \multicolumn{2}{c|}{$\begin{array}{c}\text { Media diaria } \\
\text { tuits }\end{array}$} \\
\hline Odilon Scherer & 1.715 & 2,71 \\
\hline Lluís Martínez Sistach & 691 & 1,86 \\
\hline Gianfranco Ravasi & 926 & 1,42 \\
\hline Wilfrid Napier & 884 & 1,4 \\
\hline Angelo Scola & 1.208 & 1 \\
\hline
\end{tabular}

4 "Even though you may have a million followers on Twitter, it may not necessarily mean that you are an influencer". FIEGERMAN, Seth (2012): "Former Twitter CEO Says Network Needs a Better Metric Than Follower Count". Consultado el 02/04/2013. Disponible en: http://mashable.com/2012/09/25/twitter-follower-count/ 


\begin{tabular}{|l|r|r|}
\hline Ruben Salazar & 93 & 0,85 \\
\hline Roger Mahony & 61 & 0,79 \\
\hline Timothy Dolan & 254 & 0,77 \\
\hline Jesús López Rodríguez & 313 & 0,69 \\
\hline Sean O'Malley & 363 & 0,4 \\
\hline Norberto Rivera & 218 & 0,29 \\
\hline Donald Wuerl & 55 & 0,09 \\
\hline Luis Antonio Tagle & 8 & 0,02 \\
\hline Peter Turkson & - & - \\
\hline
\end{tabular}

\subsection{Interacción}

Pero publicar muchos mensajes no es sinónimo de interacción/conversación. Como apunta Ibañez, podría ocurrir lo mismo que con la mayoría de los políticos, que sólo buscan ver quién tiene más 'tuits' y se dedican a inundar el 'timeline' actuando como 'spammers'. Según Joaquín del Moral, director de la consultora de comunicación y de marketing online Oerreeme, el problema es que muchos ven las redes sociales solo como un arma propagandística y no como un instrumento para sacar conclusiones de las conversaciones con su audiencia. Utilizan sus cuentas en Twitter como principiantes, no cuidan el feedback ni buscan generar una verdadera 'conversación' en la plataforma (citado en Ibáñez, 2011). En el caso que nos ocupa, la interacción se ha estudiado examinando los 'retuits', las menciones y las respuestas.

Un examen detenido de estas tres métricas en conjunto permite reducir a cinco el número de cardenales que realmente interactúan con los usuarios: Jesús López, Odilo Scherer, Donald Wuerl, Norberto Rivera y Wilfrid Napier. En el resto $(64,3 \%)$ predomina un modelo comunicativo jerárquizado y vertical (conocido como top-down) que emplea Twitter como una plataforma complementaria para la difusión unidireccional de mensajes (Peña y Pérez, 2011: 491). Reproducen en la red de microblogging las estrategias desarrolladas para los medios tradicionales, y no se preocupan por generar una verdadera interacción/conversación. Parece que el objetivo principal sea asociar a los cardenales con una imagen moderna, más que dotar realmente de contenidos a esas plataformas.

Si se examinan las métricas una por una, en número de "retuits's (retuitear contenidos relevantes de los usuarios a los que seguimos) sobresalen Jesús López (Santo Domingo) y el sudafricano Wuerl con más del $50 \%$ de sus mensajes, lo que "significa un buen olfato para compartir enlaces que son de interés general o por lo menos para el publico objetivo" (Bravo, 2011). Donald Wuerl vuelve a aparecer en primera posición en el apartado menciones, ya que el $30 \%$ de sus mensajes incluye por lo menos una. Le siguen el brasileño Scherer y Norberto Rivera con un $26 \%$ y un $17 \%$ respectivamente. El porcentaje de respuestas también sirve para medir la interacción ya que cuanto más alto es este indicador más interacción genera un usuario con terceros. Aquí destacan los cardenales Wilfrid Napier, Odilon Scherer, Norberto Rivera y Jesús López.

5 Retuitear contenidos relevantes de los usuarios a los que seguimos es una buena manera de aumentar la influencia de una cuenta. 
Tabla 5: Interacción. Fuente: elaboración propia

\begin{tabular}{|l|r|r|r|}
\hline \multicolumn{1}{|c|}{ Cardenal } & \multicolumn{1}{c|}{ Retuits \% } & \multicolumn{1}{c|}{ Menc. \% } & \multicolumn{1}{c|}{ Resp. \% } \\
\hline Jesús López Rodríguez & 58 & 0,14 & 12 \\
\hline Donald Wuerl & 56 & 0,29 & 11 \\
\hline Sean O'Malley & 9 & 0,05 & 2 \\
\hline Ruben Salazar & 6 & 0,11 & 10 \\
\hline Norberto Rivera & 6 & 0,17 & 18 \\
\hline Odilon Scherer & 3 & 0,26 & 19 \\
\hline Angelo Scola & 2 & 0 & 0 \\
\hline Gianfranco Ravasi & 2 & 0,04 & 0 \\
\hline Wilfrid Napier & 1 & 0,2 & 19 \\
\hline Roger Mahony & 0 & 0 & 0 \\
\hline Lluís Martínez Sistach & 0 & 0 & 0 \\
\hline Timothy Dolan & 0 & 0,06 & 0 \\
\hline Luis Antonio Tagle & 0 & 0 & 0 \\
\hline Peter Turkson & - & - & - \\
\hline
\end{tabular}

En cuanto a la media de enlaces incluidos en cada 'tuit' (hay que recordar que cuanto más alto es este número más probable es que el usuario sea una fuente de información para terceros), en este apartado sobresalen los americanos Sean O'Malley $(58 \%)$ y Timothy Dolan (38\%). Tras ellos, vuelve a aparecer el arzobispo de Washington Wuelr con un $27 \%$ de sus mensajes que incorporan un enlace. El cardenal Wuerl también es el único de los catorce perfiles encontrados que se preocupa por los 'hashtags'. Su porcentaje por mensaje es del 56\% y es un indicativo de su interés por participar en la conversación. La almohadilla o hash (\#) es importante para el posicionamiento/visibilidad de un 'tuit'. Es un recurso muy utilizado porque al añadir el símbolo '\#' a un término lo convierte automáticamente en un enlace, y al hacer click sobre él se pueden leer todos los mensajes (en el mundo) que hablan sobre ese tema. Cuando se desea hablar sobre un tema en concreto, sirve para destacar una palabra y ayudar al resto de usuarios a encontrarla fácilmente. Por lo tanto, un indicador tan alto $(27 \%)$ revela que es bastante probable que los 'tweets' del cardenal Donald Wuerl sean encontrados en una búsqueda.

\subsection{Reacciones generadas en los usuarios}

Por lo tanto, como se ha visto hasta ahora, es importante estar activo en Twitter, participar en la conversación en la que se ha convertido Internet (por ejemplo, 'retuitear' contenidos relevantes de los usuarios a los que seguimos) y aportar valor a los seguidores. Conseguir una masa crítica de seguidores interesados en los mensajes que se publican en un perfil no es el único factor a tener en cuenta. Para medir el éxito de un perfil es más eficaz examinar la repercusión que tiene en otros usuarios; como por ejemplo el número de mensajes que realmente se ven y los 'retuits' que consigue cada mensaje dentro de la red de microblogging. Algo que deja claro Evan Williams, cofundador de Twitter: "Creo que los retuits hechos por terceros son más interesantes que los seguidores [...] la simple medida de los seguidores no captura tu distribución... la métrica soñada es cuántas personas ven tu tuit y lo retuitean” (Terdiman, 2012). A 
continuación se recogen los resultados referentes a dicha métrica. Se ha contabilizado el número de mensajes y el número de veces que el mismo mensaje, tras leer el 'tuit', ha sido publicado por parte de otros usuarios (retuits).

En lo que se refiere a los mensajes que son 'retuiteados' por terceros, un valor alto significa que ha generado una reacción positiva y que el cardenal es considerado como una fuente valiosa de información por los usuarios. Como se ha mencionado antes, este indicador guarda estrecha relación con el número de veces que dichos 'tuits' son 'retuiteados' ${ }^{\prime}$. En esta métrica, que es la más importante de todas las vistas hasta el momento, es donde se nota la diferencia. En primera posición se sitúa el Cardenal Dolan, a quién sus seguidores han 'retuiteado' la totalidad de los mensajes que ha publicado (254 desde que creo la cuenta el 30 de abril de 2012). Cada mensaje del cardenal de Nueva York ha sido republicado una media de 164 veces por los cibernautas, lo que muestra la relevancia de su perfil y el valor que tienen sus aportaciones. El segundo cardenal más importante es el italiano Gianfranco Ravasi, a quien los cibernautas le han retuiteado el $96 \%$ de sus mensajes por considerarlos de interés. Cada mensaje suyo ha sido vuelto a publicar una media de 49 veces, lo que deja claro que el cardenal es considerado como una fuente valiosa de información por otros. El tercer lugar es para el arzobispo emérito de Los Ángeles Roger Mahony con una proporción de 'tweets retuiteados' por terceros del $96 \%$ y una media de 31 publicaciones por cada mensaje. Otro de los que generan mayores reacciones entre sus fieles es el cardenal Luis Antonio Tagle de Manila que, como ya se ha mencionado antes, es una estrella mediática con programa de televisión incluido. En este caso, parece que sus esfuerzos se centran en la red social Facebook (es el líder con más de 120.000 seguidores). Pero, aunque solo ha publicado ocho mensajes en la red de microblogging, todos han sido 'retuiteados' una media de 29 veces. Otros cardenales cuyos mensajes también adquieren gran repercusión son el brasileño Scherer, el colombiano Salazar, el mexicano Rivera, el americano O’Malley o el catalán Martínez Sistach.

Tabla 6: Repercusión generada. Fuente: elaboración propia

\begin{tabular}{|c|c|c|c|c|}
\hline Cardenal & $\begin{array}{l}\text { № de mensajes } \\
\text { retuiteados por los } \\
\text { seguidores }\end{array}$ & $\begin{array}{l}\text { Proporción de Tweets } \\
\text { retuiteados por } \\
\text { terceros \% }\end{array}$ & $\begin{array}{l}\text { № de veces que cada } \\
\text { mensaje es retuiteado }\end{array}$ & $\begin{array}{c}\text { Media de retuits por } \\
\text { terceros }\end{array}$ \\
\hline Jesús López Rodríguez & 78 & 25,16 & 357 & 4,58 \\
\hline Donald Wuerl & 22 & 40 & 435 & 19,77 \\
\hline Sean O'Malley & 303 & 83,47 & 3.422 & 11,29 \\
\hline Ruben Salazar & 84 & 90,32 & 3.660 & 43,57 \\
\hline Norberto Rivera & 161 & 73,85 & 731 & 4,54 \\
\hline Odilon Scherer & 1.493 & 87,06 & 33.663 & 22,55 \\
\hline Angelo Scola & 531 & 44 & 7.693 & 14 \\
\hline Gianfranco Ravasi & 891 & 96,22 & 43.698 & 49,04 \\
\hline
\end{tabular}

${ }_{6}$ No es lo mismo que los cibernautas 'retuiteen' 15 de un total de 30 mensajes de un cardenal y que cada uno se 'retuitee' 3 veces; a que solo 'retuiteen' 2 de un total de 30 mensajes de un cardenal pero que cada uno de esos 2 haya sido 'reuiteado' 800 veces. 


\begin{tabular}{|l|r|r|r|r|}
\hline Wilfrid Napier & 509 & 57,58 & 3.671 & 7,21 \\
\hline Roger Mahony & 59 & 96,72 & 1.827 & 30,97 \\
\hline Lluís Martínez Sistach & 508 & 73,52 & 3.487 & 6,86 \\
\hline Timothy Dolan & 254 & 100 & 41.653 & 163,99 \\
\hline Luis Antonio Tagle & 8 & 100 & 228 & 28,5 \\
\hline Peter Turkson & - & - & - & - \\
\hline
\end{tabular}

Por último, en lo referente a la plataforma más utilizada para publicar los mensajes hay que apuntar que el 71,4\% lo hace desde la página oficial de Twitter. El 29\% restante (el arzobispo de Sao Paulo Odilon Scherer, el arzobispo de Durban Wilfrid Napier y el arzobispo de Barcelona Lluís Martínez Sistach) prefiere su teléfono o tablet como primera opción. Asimismo, los cardenales Jesús López Rodríguez, Sean O’Malley, Gianfranco Ravasi y Timothy Dolan tienen el teléfono o tablet como la segunda plataforma más utilizada para 'tuitear'.

\section{Conclusiones}

Las redes sociales como Twitter se han convertido en una nueva forma de llegar a las personas alejadas de la Iglesia y difundir el Evangelio en un momento de crisis en que escasean las vocaciones y la religión católica pierde influencia porque no responde a las necesidades de amplios sectores de la sociedad. "Debemos transmitir la fe a jóvenes y niños. En España hemos tenido dificultades muy particulares. Luego hemos percibido cierto cansancio en los participantes habituales, que necesitan recobrar el gozo de la fe. Y tenemos que tratar de llegar a los que se reconocen agnósticos, incluso ateos", reconocía Ricardo Blázquez, vicepresidente de la Conferencia Episcopal Española. (citado en Ortiz de Latierro, 2013). Para ello, la Iglesia Católica comienza a valerse de las aplicaciones Web 2.0 y sus diferentes tecnologías como los blogs, microblogs, Facebook o Youtube, porque, entre otras cosas, amplifican el mensaje cristiano, reducen el coste de las acciones de evangelización, favorecen la cooperación entre los fieles, facilitan la difusión de información religiosa y les permite aumentar su visibilidad.

En segundo lugar, facilitan la interacción, ponen a su disposición nuevas formas de participación y permiten maneras más efectivas de organizar a los individuos (Hall y Sinclair, 2011: 59). Además, mediante el uso de las redes sociales la Iglesia Católica puede eludir a los medios de comunicación para comunicarse directamente de una manera menos distorsionada con los ciudadanos (Vergeer, Hermans y Sams, 2010: 03)

El único inconveniente es que, como se desprende de este estudio, el púlpito se sustituye por las redes pero el contenido es el mismo. En el caso de Twitter, los cardenales (un colectivo con una media de edad de 77 años) tienden a usar la herramienta más como una plataforma complementaria de difusión que como un espacio de conversación y escucha. La investigación recoge que solo el 12\% de los 117 cardenales electores que han participado en el Conclave 2013 para elegir a Francisco I han apostado por la 'ciberevangelización' e incluido Twitter en su política de comunicación. Aunque, aparentemente, esto da la sensación de ser algo positivo, todavía se trata básicamente de un modelo 'top-down', unidireccional, donde apenas se genera una verdadera 'conversación' entre el ciudadano y los cardenales. En líneas generales, parece 
sobre todo una estrategia para que los cardenales (y por tanto la Iglesia Católica) cultiven una imagen más moderna y cercana a la juventud.

\section{Referencias bibliográficas}

ABC (2012): "El primer 'tuit' de Benedicto XVI alcanza más de ochenta mil 'retuits'. 13/12/2012. Disponible en: http://www.abc.es/medios-redes/20121212/abci-twitter-papa-retuits-201212121907.html [fecha de consulta: 02 de marzo de 2013]

ACI (2013): "Miles de personas protestan a Facebook por el cierre arbitrario de una página católica de catequesis". Religionylibertad.com. Disponible en: http://www.religionenlibertad.com/articulo.asp?idarticulo=27432 [fecha de consulta: 03 de abril de 2013]

ALONSO, José; FIGUEROLA, Carlos G.; ZAZO, Ángel F. (2004): Cibermetría. Nuevas técnicas de estudio aplicables al Web. Gijón, Trea.

BAESLER, James y CHEN, Yi-Fan (2013): "Mapping the Landscape of Digital Petitionary Prayer as Spiritual/Social Support in Mobile, Facebook, and E-mail". Journal of Media and Religion. Vol. 12, $\mathrm{n}^{\circ} 1$.

BAUMGARTNER, Jody C.; MORRIS. Jonathan S. (2010): "MyFaceTube Politics : Social Networking Web Sites and Political Engagement of Young Adults". Social Science Computer Review, february, 2010. SAGE Publications. P. 24-44. [En línea]: http://ssc.sagepub.com/content/28/1/24. [Consulta el 09 de abril de 2013]

BEDOYA, Juan G. (2012): "Europa, la nueva tierra de misión”. En: El País 21/02/2012. Disponible en: http://sociedad.elpais.com/sociedad/2012/02/20/actualidad/1329769985_653957.html [fecha de consulta: 02 de abril de 2013]

BECKFORT, James (2003): Social Theory and Religión. Cambridge University Press.

BRAVO, Carlos (2011): Por qué 10.000 followers en Twitter no te hacen más influyente. [En línea]: http://www.marketingguerrilla.es/porque-10-000-followers-entwitter-n... [Consulta el 02 de abril de 2013].

CAMPBELL, Heidi (2010): When religion meets new media. Routledge, New York, p. 213.

CARAVACA, Toñi (2013): "Yo os bendigo, en el nombre de Twitter y Facebook". El Mundo, 8 de febrero. Disponible en: http://www.elmundo.es/elmundo/2013/02/08 /andalucia/1360354649.html [fecha de consulta: 02 de abril de 2013]

CENTRO DE INVESTIGACIONES SOCIOLÓGICAS (CIS) (2008): Datos del estudio 2.752 de 2008

CENTRO DE INVESTIGACIONES SOCIOLÓGICAS (CIS) (2013): Datos del estudio 2.981 de Marzo 2013

CISCO (2011): Cisco Connected World Technology Report (Segundo informe anual) 2011. [En línea]: http://www.cisco.com/web/ES/about/press/2011/11-09-21Cisco_Connected_World Report_2011_Parte_I.html [Consulta el 02 de abril de 2013]. 
COLEGIO CARDENALICIO (2013): Disponible en: http://www.vatican.va/news services/press/documentazione/documents/cardinali_statistiche/cardinali_statistiche_eta_it.html [fecha de consulta: 02 de abril de 2013]

COPE (2013): "Programa La Tarde", 21 de febrero de 2013.

CORDERO MORALES, Fernando (2008): “Análisis y perspectivas de la información religiosa especializada". Ámbitos, 17, pp. 75-87

DIARIO 20 MINUTOS (2010): "Instalan en la catedral Santiago velas que se encienden mediante Internet y sms". Disponible en: http://www.20minutos.es/noticia/688937/0/velas/internet/sms/ [fecha de consulta: 02 de abril de 2013]

DÍAZ, Lorenzo (1993): La radio en España 1923-1993. Madrid, Alianza Editorial.

FIEGERMAN, Seth (2012): "Former Twitter CEO Says Network Needs a Better Metric Than Follower Count". Disponible en: http://mashable.com/2012/09/25/twitter-follower-count/ [fecha de consulta: 02 de abril de 2013]

FUNDACIÓN TELEFÓNICA (2012): La Sociedad de la Información en España (SIE). Barcelona, Ariel. [En línea]: http://elibros.fundacion.telefonica.com /sie11/aplicacion_sie/ParteA/pdf/SiE_2011.pd [Consulta el 02 de abril de 2013]

GIL DE ZÚÑIGA, Homero; PUIG-I-ABRIL, Eulàlia; \& ROJAS, Hernando (2009): "Weblogs, traditional sources online and political participation: an assessment of how the Internet is changing the political environment". New Media Society, 11, 553-574.

GÓMEZ GARCÍA, Salvador (2009): "Entretenimiento y fe en las ondas. Las emisiones religiosas de Radio Nacional de España durante el primer franquismo (19391959)". Estudios sobre el Mensaje Periodístico, vol. 15, pp. 261-276. Madrid, Servicio de Publicaciones de la Universidad Complutense.

GUEORGUIEVA, Vassia (2008): "Voters, MySpace, and YouTube : The Impact of Alternative Communication Channels on the 2006 Election Cycle and Beyond". Social Science Computer Review, Volume 26, Number 3, pp. 288-300.

IBÁÑEZ, Creu (2011): “Elecciones 20N. ¿Candidatos políticos o 'spammers' en Twitter?”. En: lainformación,com. [En línea]: http://noticias.lainformacion.com/espana/elecciones-20n-candidatos-politicos-o-spammers-en-twitter_fYwKXIfOOVIj BwOpq0TZ1/ [Consulta el 02 de abril de 2013]

LORENTE CANO, Mónica (2011): "Social TV en España: concepto, desarrollo e implicaciones". Cuadernos de gestión de información, pp. 55-64

MANCILLA, Alma (2011): "La representación de la religión en el espacio público entre los líderes religioso minoritarios en Mexico. Entre deseo de reconocimiento y exigencias de participación". Revista de Estudios Sociales, $\mathrm{n}^{\circ}$ 39. Universidad de los Andes, pp. 80-94 [En línea]. http://res.uniandes.edu.co/view.php/697/view.php [Consulta el 02 de abril de 2013].

MÚJICA, Jorge Enrique (2013): "Reacciones y contestaciones al Papa en Twitter en un estudio". Disponible en: http://evangelidigitalizacion.blogspot.com.es/2013/01 
/un-mes-del-papa-en-twitter-reacciones-y.html. [fecha de consulta: 02 de abril de 2013]

ORTIZ DE LATIERRO, Zuriñe (2013): "En manos de Francisco". El Correo, 31 de marzo, pp. 2-4.

PEÑA, Simón y PÉREZ DASILVA, Jesús (2011): “Internet y participación ciudadana en las elecciones autonómicas de Euskadi 2009". Actas del XVII Congreso Internacional de la SEP. Valladolid, pp. 481-500.

PÉREZ LATRE, Francisco Javier (2012): “Los medios y la religión en el contexto de la nueva evangelización". Revista Scripta Theologica, vol 44, pp. 169-185. Pamplona.

POU AMÉRIGO, María José (2008): "El hecho religioso y su tratamiento periodístico: limitaciones y dificultades". Estudios sobre el Mensaje Periodístico, vol. 14, pp. 561-573. Madrid, Servicio de Publicaciones de la Universidad Complutense.

RODRÍGUEZ, Ma del Mar et al. (2013): "Información y comunidad para madres en Internet: análisis de las principales webs de bebé en España". Comunicación y Sociedad. Vol. XXVI, nº 1 , pp. 22-46.

ROJO GARCÍA, José María (2006): "Evangelización y medios de comunicación, Hacia una radio misionera". Reflexiones, Santo Domingo, pp. 49- 61.

RTVD (2008): "Los representantes de España en el Congreso Mundial de Radios Católicas coinciden en la "misión evangelizadora" de estos medios". Disponible en: http://www.rtvd.org/Radios\%20Catolicas.htm [fecha de consulta: 02 de abril de 2013]

SAID, Elías y ARCILA, Carlos (2011): "Líderes de opinión en Colombia, Venezuela e Irán. El caso de los 20 usuarios más vistos en Twitter". Comunicación y Sociedad, vol. XXIV, $\mathrm{n}^{\circ} 1$, pp. 75-100.

SÁNCHEZ REDONDO, María Isabel (2001): Historia de la COPE (1959. 1983). Una radio diferente. Valencia, Fundación Universitaria San Pablo CEU.

SUDULICH, Laura et al. (2010): "Me too for web 2.0? Patterns of online campaigning among candidates in the 2010 UK general election". Internet, Politics, Policy 2010: An Impact Assessment conference, organized by the Oxford Internet Institute, and held at St. Anne's College, Oxford, UK, on September 16-17, 2010. [En línea]: http://microsites.oii.ox.ac.uk/ipp2010/system/files/IPP2010_Sudulich_Wall _Jansen_Cunningham_Paper.pdf [Consulta el 16 de abril de 2013].

TASCÓN, Mario y QUINTANA, Yolanda (2012): Ciberactivismo. Las nuevas revoluciones de las multitudes conectadas. Madrid, Catarata.

TERDIMAN, Daniel (2012): Twitter founder says influence is in retweets. NFL player shows why. [En línea]: http://news.cnet.com/8301-1023_3-57520148-93/twitter-founder-says-influence-is-in-retweets-nfl-player-shows-why/ [Consulta el 02 de abril de 2013].

TNS (2012): Estudio Digital Life. Disponible en: http://www.slideshare.net/imartinmorales/estudio-mobillife-de-tns [Consulta: 02/04/13]. 
THE COCKTAIL ANALYSIS (2012): Observatorio de redes sociales. IV Oleada, abril de 2012. [En línea]: http://www.slideshare.net/TCAnalysis/4-oleada-observatorio-de-redes-sociales [Consulta el 02 de abril de 2013].

VANINNI, Giovanni (2013): "Un tweet color porpora". Revista Popoli, 27 de enero. [En línea]: http://www.popoli.info/EasyNe2/Primo_piano/Un_tweet_color_porpora.aspx [Consulta el 02 de abril de 2013].

VERGEER, Maurice; HERMANS, Liesbeth; \& SAMS, Steven (2010): “The voter is only a tweet away micro blogging during the 2009 European Parliament election campaign in the Netherlands". Paper 13. III Annual Political Networks Conference [En línea]: http://opensiuc.lib.siu.edu/pnconfs_2010/13 [Consulta el 08 de abril de 2013].

WILLIAMS, Rhys H. (2003): "Religious Social Movements in the Public Sphere: Organization, Ideology and Activist". Handbook of the Sociology of Religion, pp. 315-330. Cambridge University Press. 